\title{
Learner Autonomy through the Adoption of Open Educational Resources Using Social Network Services and Multi-media E-textbooks
}

\author{
Masumi Hori* \\ NPO CCC-TIES, Japan \\ Seishi Ono \\ NPO CCC-TIES, Japan \\ Shinzo Kobayashi \\ SmileNC \& Co., Japan \\ Kazutsuna Yamaji \\ National Institute of Informatics, Japan \\ Toshihiro Kita \\ Kumamoto University, Japan \\ Tsuneo Yamada \\ The Open University of Japan, Japan
}

\begin{abstract}
With the development of social network services (SNS) on the Internet, the world has gained access to vast amounts of information resources, allowing people to carefully research and select what they need and want to share with others. The main idea behind open educational resources (OER) is providing freely accessible and openly licensed documents, which fits well with the online learning system using SNS. However, the gap between higher education and social network media in relation to shared activities and OER use remains a challenge. The main reason for this is that teachers lack knowledge of mutual assistance and the skills to use OER. Teachers are dissatisfied with having to use others' resources, which indicates that the problem lies in teachers' psychological conflicts and technical capabilities. Our learning platform, Creative Higher Education with Learning Objects (CHiLO), is based on e-textbooks and aims to develop a flexible learning environment. The CHiLO e-textbooks were developed with a completely new design that considered large-scale online courses, such as open online courses. The core component of CHiLO is the CHiLO Book, which is created in EPUB3 format and has media-rich contents, including graphics, animations, audios and embedded videos. Our set of experimental outcomes shows that CHiLO, which includes not only Web services but also e-textbooks, is easy for teachers to handle.
\end{abstract}

Keywords: open education resources, open education, e-books, massive open online courses, social network services, open badges

\footnotetext{
${ }^{*}$ Corresponding author. Email: hori@cccties.org
} 


\section{Introduction}

The development of social network services (SNS) has made large amounts of information resources accessible and has also made it easier for people to choose and share resources. Several ideas for online education using SNS have been proposed. One of these which fits the online learning system well is open educational resources (OERs), which are freely accessible and openly licensed documents.

However, substantial gaps exist between higher education and social network media, and between teachers and students, with regard to sharing activity and OER use. These gaps are mainly due to the challenges associated with the lack of mutual assistance and OER skills among teachers and their dissatisfaction with using resources created by someone else. Therefore, these problems can be said to stem from teachers' motivation, technical expertise, and psychological conflicts. Meanwhile, a growing number of higher education students are establishing various online relationships and extensively sharing knowledge through open network communities, such as SNS, blogs, and wikis. It is now impossible to meet students' interests using only current learning management systems hosted by institutions (Sclater, 2008).

Another online education idea involves the use of e-textbooks. Based on the e-book EPUB3 format, the International Digital Publishing Forum (IDPF) proposed the EDUPUB format to meet the requirements of next-generation learning content (IDPF, 2014). However, most e-book readers do not currently support the media-rich functions of the EDUPUB format, such as embedded videos, JavaScript compliance, and JavaScript Object Notation (JSON).

Creative Higher Education with Learning Objects (CHiLO) aims to provide a comprehensive open-network learning system using existing technologies, e-textbooks in EPUB3 format, and various learning resources, including OER, on open network communities, such as SNS.

The basic outline of this paper is as follows. First, it provides a brief overview of the CHiLO architecture, and then presents our methodology and experiment's results. Next, it discusses the findings and, finally, offers concluding comments.

\section{New Architecture Using E-textbooks in Open Network Learning}

Goodyear (2005) described networked learning as a learning model in which information and communications technology are 'used to promote connections between one learner and other learners; between learners and tutors; between a learning community and its learning resources.' To realize the learning model, the learning resources in the model should be aggregated, remixed, repurposed and shared (Kop, 2011). 
A learning method using e-textbooks provides a new way of learning that is adapted to the network learning model. The advent of the EPUB3 format means that e-books now include media-rich and interactive content. The IDPF (2011) stated:

The EPUB specification is a distribution and interchange format standard for digital publications and documents. EPUB defines a means of representing, packaging, and encoding structured and semantically enhanced web content - including HTML5, CSS, SVG, images, and other resources - for distribution in a single-file format.

Thus, the EPUB3 format has greater sourcing flexibility. In the field of education, learning materials in the EPUB3 format can easily be repurposed by tutors, adapted to improve learning outcomes, and used to avoid vendor lock-in (Belfanti, 2014).

Our CHiLO was created based on large-scale online courses in comprehensive online learning systems using EPUB3 e-textbooks in an open-network environment. CHiLO creates learner communities in large-scale online courses to promote active connections among learners through shared open learning resources.

CHiLO consists of four elements: CHiLO Book, CHiLO Lecture, CHiLO Badge, and CHiLO Community (Figure 1).

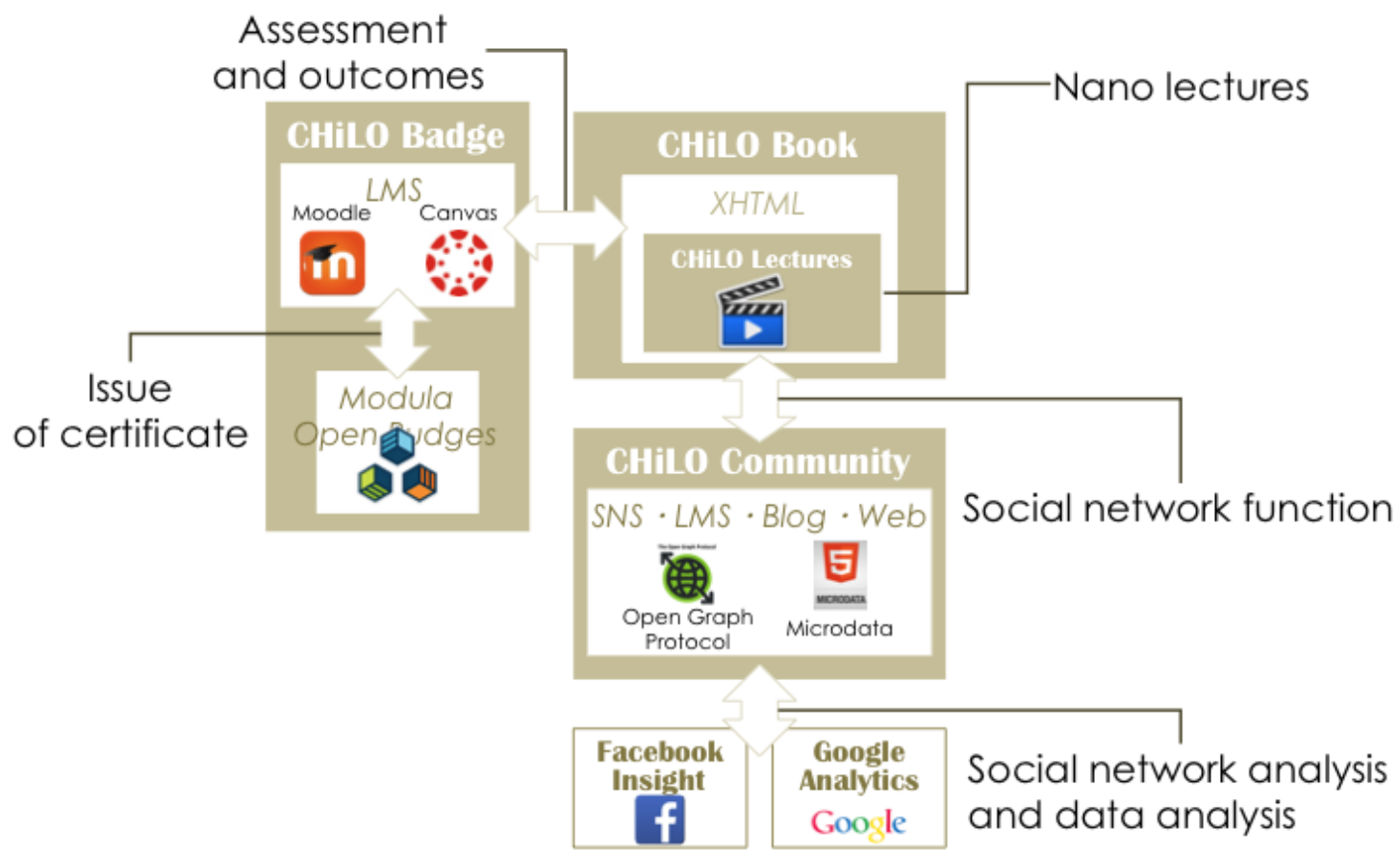

Figure 1 Conceptual diagram of CHiLO 


\section{CHiLO Book and CHiLO Lecture}

The core component of CHiLO is CHiLO Book, which is created in EPUB3 format and contains media-rich content, including graphics, animation, audio, and embedded video.

CHiLO Lecture consists of videos with scripts, quizzes, and other learning materials. Videos are one-minute nano-lectures. This concept came from an experiment that revealed that most online learners' viewing time is approximately one minute (Figure 2) (Hori et al., 2013).

A CHiLO Lecture is equivalent to one section in a traditional textbook, and a CHiLO Book consists of approximately ten CHiLO Lectures. A standard CHiLO course, which is comparable to a traditional university course with one academic credit, consists of ten CHiLO Books.

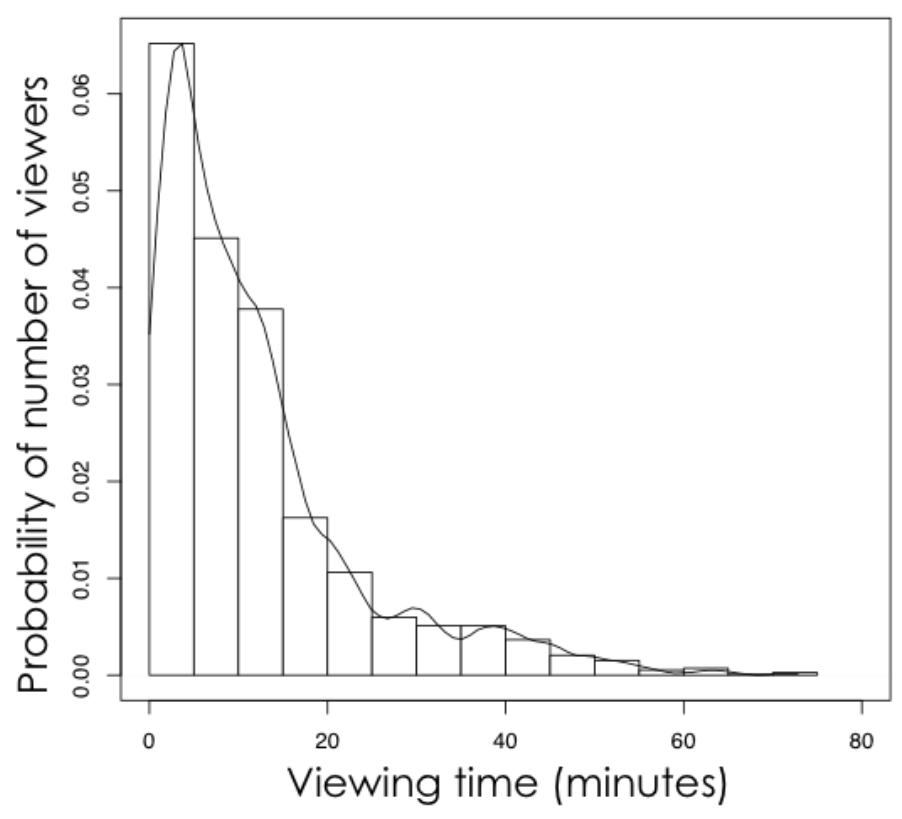

Figure 2 Learner viewing time (Hori et al., 2013)

\section{CHiLO Badge}

It is difficult to perform indirect assessments of, for example, learning time and academic workload in large-scale online courses. Although CHiLO adopts a direct assessment approach for learning outcomes, completion of a CHiLO course is measured in standard course hours, which correspond to academic credits.

Whenever learners complete a CHiLO Book, they receive a CHiLO Badge, which is a simple mechanism for successful outcome assessment in CHiLO. When a tutor wishes to check a learner's progress, he/she asks the learner to present his/her CHiLO Badges, thus removing the need to confirm using indirect assessment tools, such as grade books 
and tracking past results, or test scores. CHiLO Badges are based on Open Mozilla badges (see http://www.openbadges.org).

\section{CHiLO Community}

A CHiLO Community consists of many learners and a few tutors, known as 'connoisseurs'. These tutors act as substitutes for teachers. A learner who has studied and completed CHiLO Books in a specific field can become a connoisseur. The connoisseur and learner are on an equal footing, so a connoisseur often exchanges information with learners in their community.

In the CHiLO Community, learners do not learn from a tutor but rather learn on their own using CHiLO Books as a learning resource. In this way, learners are constantly required to find suitable $\mathrm{CHiLO}$ Books within the community. The CHiLO Community provides methods for discovering, sharing, aggregating and repurposing CHiLO Books for learners.

The first of these methods is the Open Graph Protocol (OGP), which was originally created for Facebook (see https://www.facebook.com/facebook/info), but is also available via other web services. OGP allows any webpage to become a rich object in a social graph (see http://ogp.me). Webpage contents can be readily shared by converting them to OGP.

The second method is microdata. Microdata, which is the Web Hypertext Application Technology Working Group's HTML specification, is a mechanism for adding machine-readable annotations to documents, so certain tools can extract trees of name-value pairs from the document (WHATG, 2014). It is easy to provide information to any user through search engines using metadata in a webpage.

A CHiLO Book, including an XHTML file with OGP and microdata, is connected by dedicated referral servers. Metadata tags for OGP and microdata in the CHiLO Book contain the title, a description, where to download it from, and a thumbnail index. Learners can easily search the contents of any CHiLO Book using Web search engines, such as Google, Yahoo or Bing, via a referral server. 


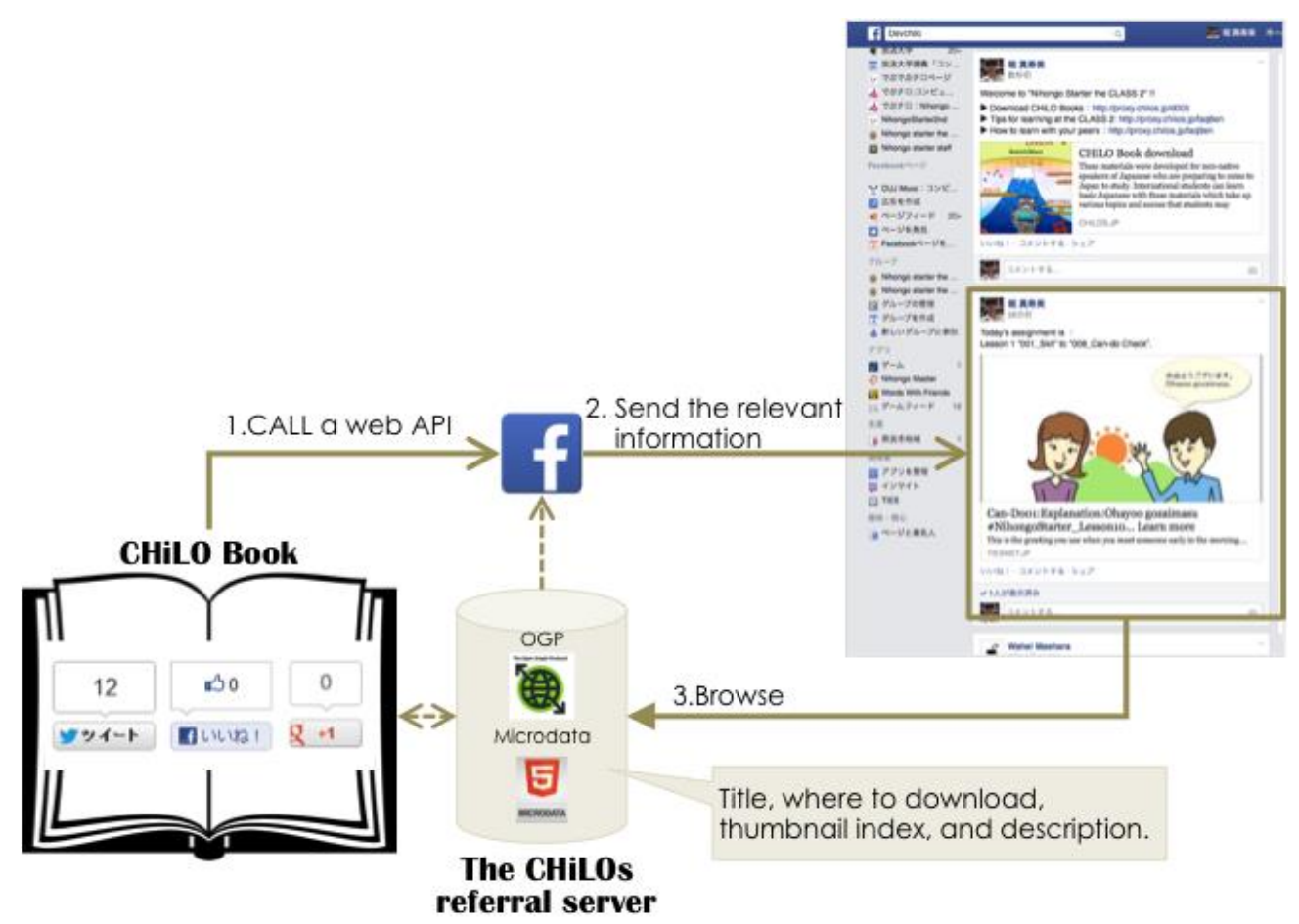

Figure 3 The OGP mechanism in a CHiLO Book

Figure 3 shows an example of OGP instructions in a CHiLO Book. A learner posts an article, comment or question on content to an SNS, such as Facebook, Twitter, or Google Plus, or his/ her own SNS via a CHiLO Book. A social network share button that links to a Web application programming interface (API) is embedded in an XHTML file within a CHiLO Book. When the learner clicks 'post', the CHiLO Book sends the relevant information from the CHiLO referral server to the SNS, along with the content to be shared. When another learner sees the post, he/she can access the information contained in the CHiLO Book. Thus, learners in the CHiLO Community can obtain the learning materials they need through CHiLO Books and SNS.

\section{Comparison with EDUPUB}

Framing EDUPUB as an e-textbook enables online assessments and learning analytics and includes EPUB3's extended Learning Tools Interoperability, IMS Caliper Analytics, and IMS Question and Test Interoperability (Mattson et al., 2014).

CHiLO fulfils the same purpose as EDUPUB without using EDUPUB specifications. For example, the CHiLO Badge allows online assessment, whereas the CHiLO Community provides analytics, social network analysis and data analysis.

EDUPUB is implemented in JSON, but most e-book EPUB readers do not support JSON. One reader that does support JSON is Readium, which is an open source EPUB 
reader developed by the IDPF. However, one of Readium's disadvantages is that it currently does not support mobile devices, such as smartphones and tablets. CHiLO Books offers a realistic solution by combining an e-book reader and a Web browser.

\section{Results of Demonstration Experiment}

\section{Experimental methodology}

Cyber Campus Consortium TIES (NPO CCC-TIES) developed the Japanese-language study content, known as 'Nihongo Starter A1' (NS A1), in partnership with the Open University of Japan (OUJ) and the Japan Foundation.

We commenced a demonstration experiment of NS A1, delivered as an OUJ massive open online course (MOOC), in November 2013, in cooperation with OUJ and the Japan Massive Open Online Course Consortium (JMOOC). JMOOC is 'an organization that was formed in 2013 with the cooperation of Japanese universities and businesses that aims to spread and magnify Japanese MOOCs throughout the country' (JMOOC, 2013).

NS A1 is intended to be a beginner's course. It consists of ten CHiLO Books for non-native Japanese speakers based overseas who would like to study in Japan. One CHiLO Book consists of a lesson with 20-25 lecture videos and two to three online exams. One CHiLO Badge is issued after each online exam is passed.

The experimental approach was as follows. The NS A1 and a ten-volume CHiLO Book set was delivered to commercial e-book sites, such as the Google Play Bookstore and the iTunes Store, at no charge. Simultaneously, two new Facebook groups named Class 1 and Class 2 were created for NS A1 to establish the CHiLO Community. Learners from each class applied and could become a connoisseur, such as a Japanese-language teacher.

Class 1 and Class 2 were opened for four weeks each. Class 1 was held from April 14, 2014 to May 11, 2014 and Class 2 from June 2, 2014 to June 29, 2014. The locations at which the CHiLO Book sets could be downloaded were posted to the Facebook groups every week. Connoisseurs took care of all the learners' inquiries. A CHiLO Badge was issued after learners completed each CHiLO Book and each NS A1 lecture. Every week, to facilitate learning, all reference pages were posted in Class 1, with further significant reference pages posted in Class 2.

\section{Experimental results}

Figure 4 shows the learner activities, such as the number of CHiLO Book downloads, the video audiences, the completed quizzes, and the badge earnings. Because the CHiLO Books were delivered to commercial e-book sites, the total number of downloads was more than the number of participants in the course. 
The experiment included 1,279 participants from 71 countries, including the United States, Mexico, Colombia, Malaysia, Australia, Thailand and Vietnam. Class 1 included 440 participants, and Class 2839.

After the first week of Classes 1 and 2, the number of video views sharply decreased. Although 388 unique users downloaded the first volume of Class 1's CHiLO book and 699 unique users downloaded Class 2's book, only $44 \%$ and $48 \%$ of the users viewed the videos, respectively. After downloading the CHiLO Book, many participants did not watch the videos. This shows that CHiLO Books may be difficult for some learners to operate because they must manage an e-book reader and a Web browser at the same time. As a result, only two participants in Class 1 and nine in Class 2 completed NS A1 and received a final CHiLO Badge.
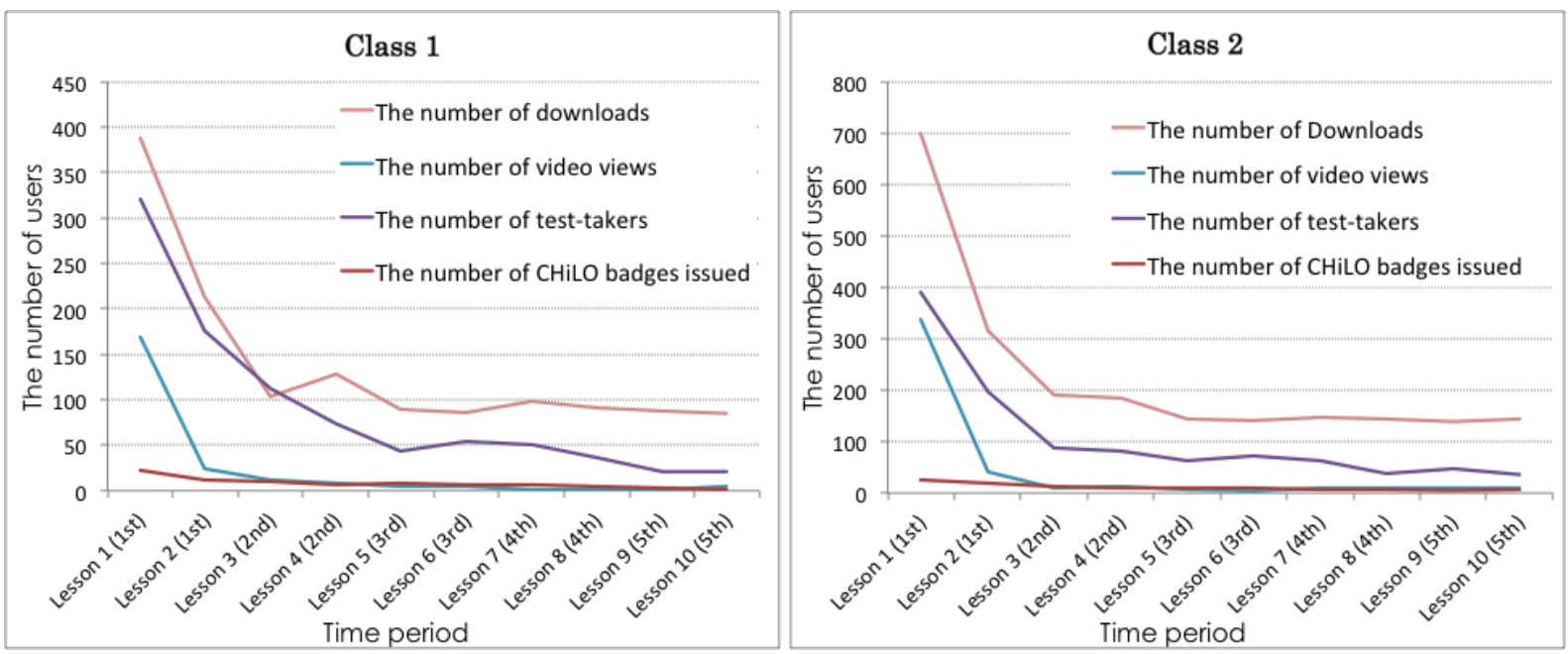

Figure 4 Leaner activities

Figure 5 shows the number of unique users who attempted online exams on a weekly basis. Although those who posted reference information tended to attempt online exams in both Classes 1 and 2, it was clear that many learners studied at their own pace. 

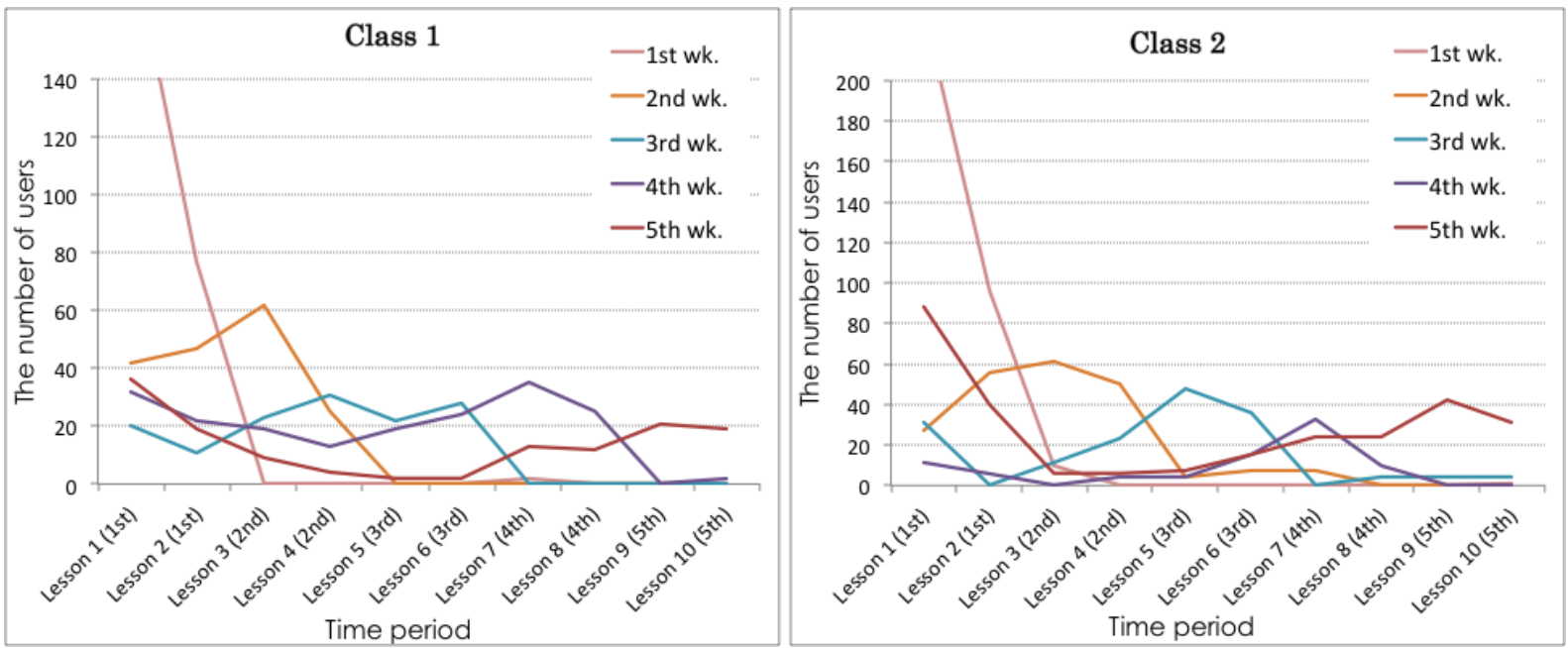

Figure 5 The number of participants who attempted online exams

In Figure 6, it can be seen that the number of learners increased steadily over time. In Class 2, a sharp rise in new participants was seen in the final three days of the course. The results show that a significant number of participants learned at their own pace.

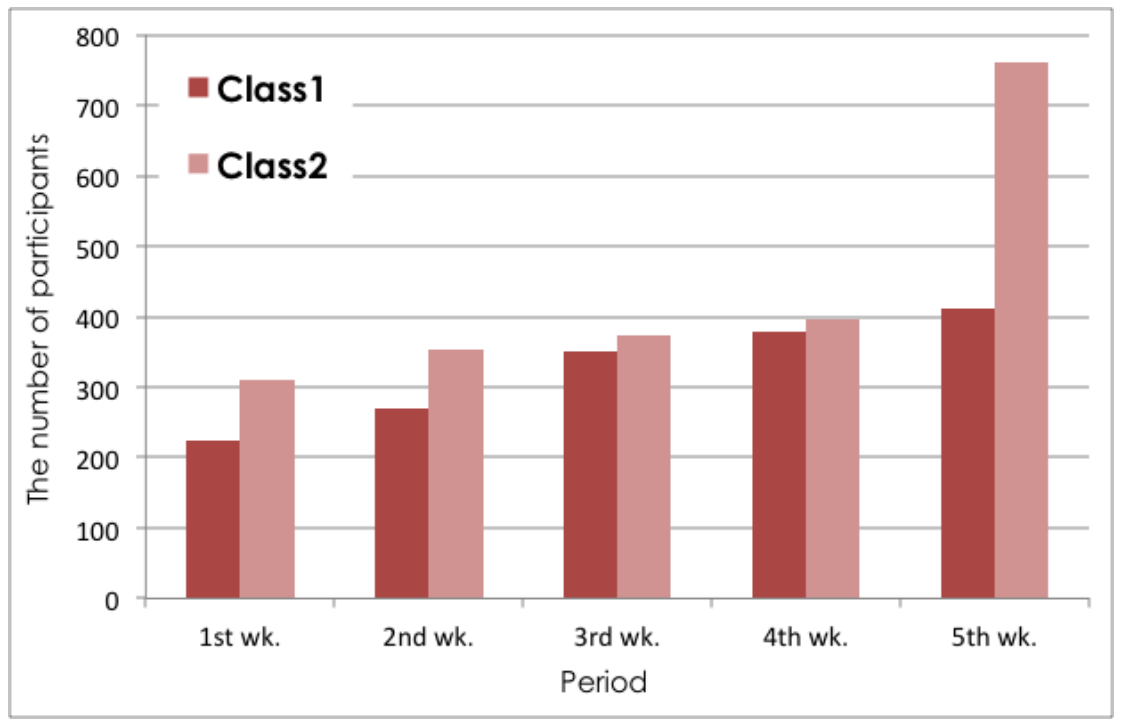

Figure 6 Number of participants joining the course

Figure 7 shows the number of CHiLO Book downloads among the participants. The first volume of NS A1 was the most commonly downloaded book every week. This demonstrates that participants who joined in the middle of the semester still utilized the first volume in the series. 

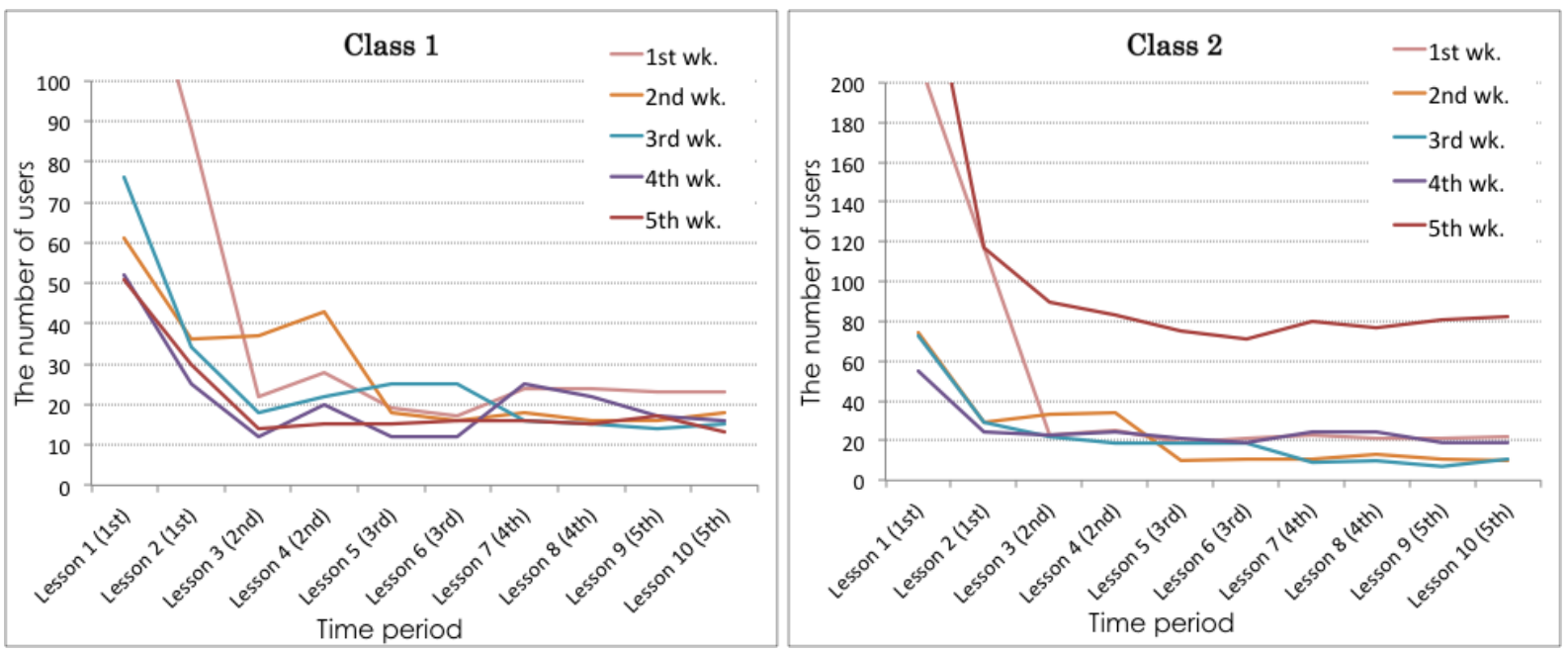

Figure 7 Number of participants downloading CHiLO Books

The participants' video views are shown in Figure 8. A tendency similar to the results given above was observed, with the greatest number of views seen early in the course.
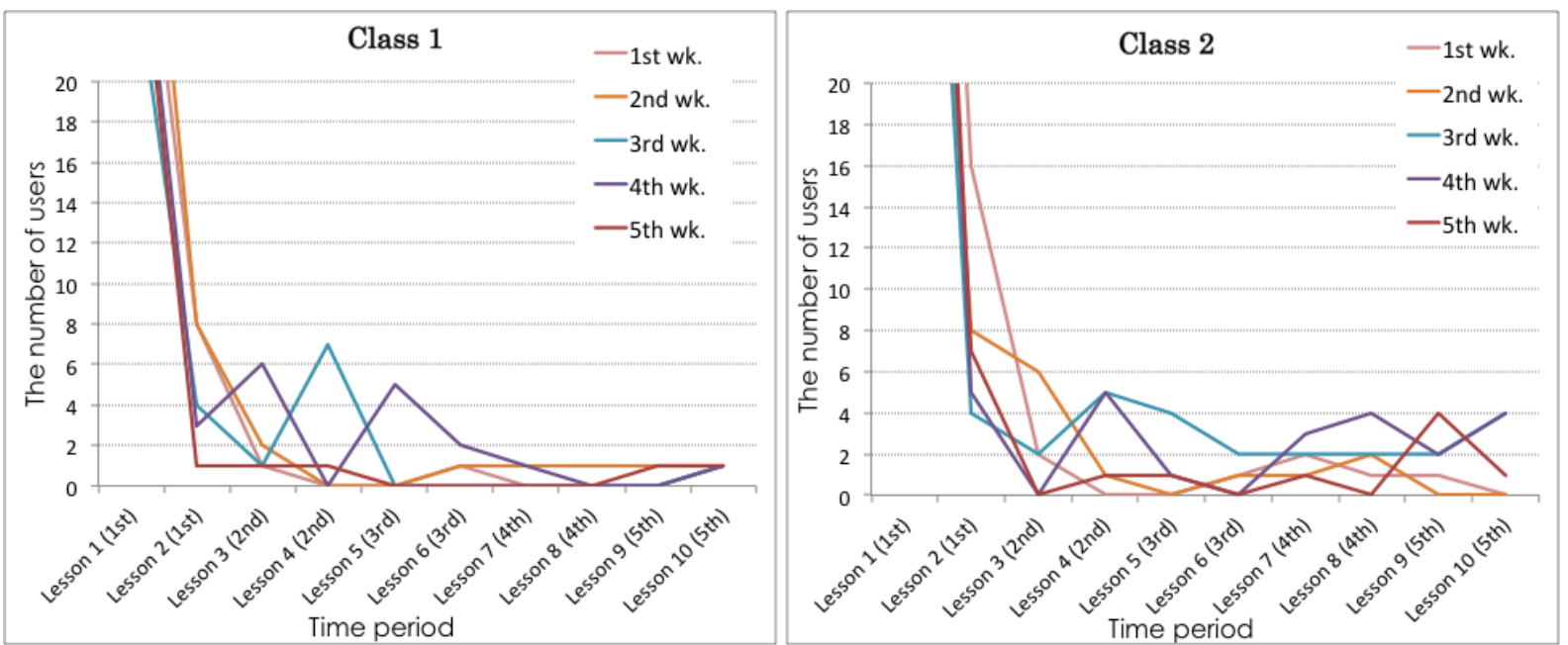

Figure 8 Number of participants viewing videos

\section{Discussion of Experimental Results}

Many people asked questions about how to view the e-books or complained about the posts on the Facebook group pages. For example, some users said, 'I am in Nepal. I am not able to download CHiLO Books'; 'iTunes and Google Play are not accessible from Egypt'; and 'I downloaded a CHiLO Book, but the EPUB Reader won't launch'. These 
statements indicate that Google Play and iTunes are not accessible in some countries or regions and that not all learners are familiar with using e-books.

As seen in Figure 4, less than half of the learners who downloaded the first e-book did not view the videos. Comments from the learners included the following: 'I would like to learn with a video-embedded EPUB e-book'; 'It was not good to find that some content was not embedded'; and 'It took 25 minutes to register with Google Play, change the cookie settings, instal the EPUB reader, and now I can see the video! ... but, there were no videos embedded'. Complaints such as these are real issues associated with the usability of non-stand-alone e-books that require an external Web browser to be used.

Nevertheless, the activity of users who could use the e-books was interesting. They tended to use the learning materials (video, online exams, e-books) from previous lessons and the newer materials they were notified about by the connoisseurs according to the course schedule. This may have occurred because some students learned autonomously without needing a predefined schedule and because learners who joined the course later began with the first lesson. This situation could be unique to packaged online courses in which users can download the complete course content and learn at their own pace.

One of the merits of using Facebook was that the learners' progress through the e-books was facilitated by the connoisseurs' postings about each week's lesson. Although information-sharing between learners on the SNS was not confirmed, one tutor's post from the second week of Class 1 was shared among many Facebook users. Because of this, the number of page views increased temporarily, but the number of learners did not increase.

\section{Conclusion}

CHiLO is an e-book-based framework in open networks that aims to enhance connections between learners, learning resources, and the community. Because the number of applications and devices that are EPUB3-compliant is limited, we designed $\mathrm{CHiLO}$ to use an external browser for viewing videos and taking online exams.

Users complained about issues with downloading e-books and the unavailability of embedded videos. Interestingly, learners who could use the e-books tended to use learning materials from previous lessons. This tendency could indicate that autonomous learning occurred and could be regarded as a benefit of the e-book-based framework compared with those found in traditional web-based learning environments. However, the framework is somewhat problematic because it does not yet allow learners to share their learning experiences with others whose progress is considerably different. This problem is worth considering in future work. 
To address users' complaints, we developed a Web version of CHiLO Books that is automatically created from the same data files as the EPUB3 versions. This Web version enables us to provide quick and easy learning materials for those who prefer to learn using Web browsers. Along with the EPUB3 version of CHiLO Books, we hope to improve usability by refining our CHiLO framework.

\section{References}

Belfanti, P. (2014). What is EDUPUB. Retrieved August 1, 2014, from http://www.imsglobal.org/edupub/WhatisEdupubBelfantiGylling.pdf

Goodyear, P. (2005). Educational design and networked learning: Patterns, pattern languages and design practice. Australasian Journal of Educational Technology, $21(1), 82-101$.

Hori, M., Ono, S., Kobayashi, S., Yamaji, K., \& Kita, T. (2013). Peer-to-peer learning on large scale online courses: Focusing on lurkers. Proceedings from the 6th International Conference on e-Learning and Innovative Pedagogies, November 14-15, 2013, Madrid, Spain. Retrieved August 1, 2014, from http://ubi-learn.com/_uploads/FULLPROGRAM.pdf

International Digital Publishing Forum (IDPF) (2011). EPUB3 Overview: Recommended specification 11 October 2011. Retrieved August 1, 2014, from http://www.idpf.org/epub/301/spec/epub-overview.html

International Digital Publishing Forum IDPF (2014). EPUB 3 EDUPUB Profile draft specification, 28 May 2014. Retrieved August 1, 2014, from http://www.idpf.org/epub/profiles/edu/spec/

Japan Massive Open Online Courses (2013). About JMOOC. Retrieved August 1, 2014, from http://www.jmooc.jp/en/about/

Kop, R. (2011). The challenges to connectivist learning on open online networks: Learning experiences during a massive open online course. The International Review of Research in Open and Distance Learning, 12(3), 19-38

Mattson, L., Smythe, C., Vickers, S., Anderson, T., Vento, C., \& Nayak, P. (2014). Using IMS caliper, question \& test interoperability (QTI) and learning tools interoperability (LTI) with EPUB3: EDUPUB best practices. Retrieved August 1, 2014, from http://www.imsglobal.org/edupub/EPUB3QTILTICaliper_BestPracticesvd8.pdf

Sclater, N. (2008). Web 2.0, personal learning environments, and the future of learning management systems. EDUCAUSE Center for Applied Research Bulletin, Volume 2008, 13, 1-13. 
The Web Hypertext Application Technology Working Group (WHATWG) (2014). HTML Living standard, Retrieved August 1, 2014, from http://www.whatwg.org/specs/web-apps/current-work/multipage/ 